\title{
Automobile safety technology and its improvement
}

\author{
Xiao-Yang Wang ${ }^{1}$, Yi-Hang Jiang ${ }^{1}$,Ren-Bo Li ${ }^{2}$ and Ming-Zhang Chen ${ }^{3}$ \\ ${ }^{19}$ Wuhan University of Technology, Wuhan, China \\ 1Wuhan University of Technology, Wuhan, China \\ ${ }^{2}$ Wuhan University of Technology, Wuhan, China \\ ${ }^{3}$ Wuhan University of Technology, Wuhan, China
}

\begin{abstract}
With the development of society, and the sharp increase in car sales, traffic accidents have become increasingly frequent and serious .At the same time, with the rapid development of science and technology, automotive technology has also been rapidly improved, automotive safety technology gradually involved in all aspects of the car. This paper introduces the main automotive safety technology at present, proposes a kind of airbag system, and describes its specific working methods.
\end{abstract}

\section{Introduction}

With the continuous improvement of the living standards of the society and the people, the automobile industry as an economic pillar is also developing rapidly. In recent years, the car ownership in China has been growing rapidly. Cars are no longer luxuries. According to scientific statistics, China has exceeded 140 million vehicles in 2014, accounting for $2 \%$ of the world's total. As early as 2013, the number of vehicles had broken 137 million mark, which is about 5.7 times the size ten years ago, as shown in Figure 1. When the automobile has brought efficient development to our society and rapid growth to our economy, the widespread use of the automobile has also brought us some negative effects, such as traffic congestion, environmental pollution, traffic accidents. Among them, the impact caused by traffic accidents is the most direct, causing the biggest loss, making the most widely damage.

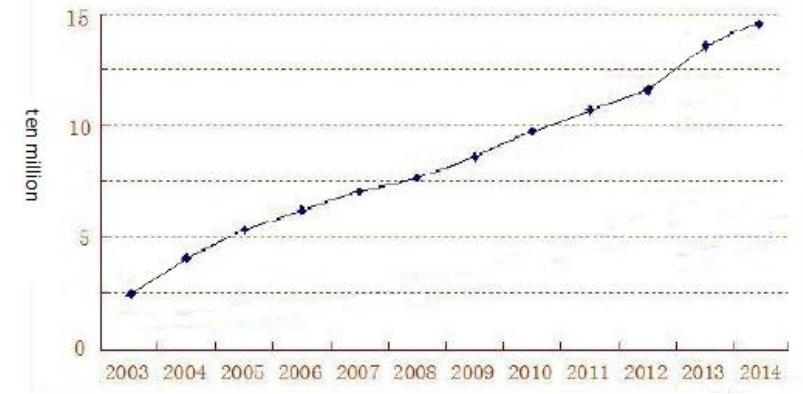

Figure 1. The number of Chinese cars

With the continuous popularization of cars, the number of cars in China is constantly increasing, the speed of cars is increasing, and traffic accidents are becoming more frequent. According to the data on the cause of death of sick residents by the health department, 166,906 people died of motor vehicle accidents in 2012, more than double that of a decade ago, as shown in Figure 2.If appropriate measures are not taken, traffic accidents will be the third leading cause of global diseases and injuries by $2020^{[1]}$. In addition, according to the relevant reports, the economic losses of automobile traffic accidents accounted for 1\% of GDP in low-income countries and $2 \%$ in high-income countries. Global car traffic accident damage is estimated at $\$ 518$ billion per year $^{[2]}$. Therefore, the automotive safety technology has become one of the themes of the development of international automotive high and new technology.

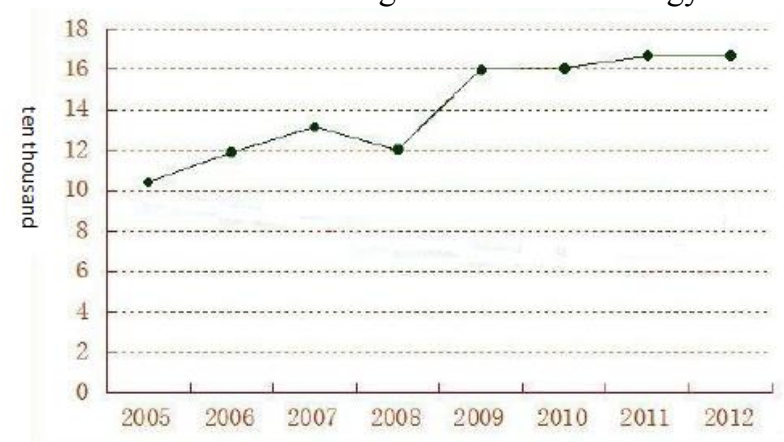

Figure 2. Death toll of motor vehicle accidents

Since 1980s, with the rapid development of computer technology and the development of multi rigid body and finite element software, computer simulation technology has been widely used in the study of vehicle passive safety, pedestrian and occupant protection and related issues. Such as the deduction of mechanical analytical formula of typical body structure components by Wierzbick. $T$ et al. The calculated results are in good agreement with the experimental data, providing 
sufficient basis for the conceptual design of automobiles. Through the collision test and computer simulation analysis, the vehicle passive safety problems, such as frontal and side crashworthiness, occupant restraint system matching, pedestrian protection and so on, are deeply studied by Huang.M ${ }^{[3]}$. et al. As shown in Figure 3.Car manufacturers in developed countries have mastered the positive development design technology for the passive safety of vehicles. In addition, the simulation software MADYMO self-developed by TNO company in the Netherlands after joining the AUTO DOE module, successfully achieved the parameter optimization and performance control of the occupant restraint system, and is widely used in automotive passive safety development.
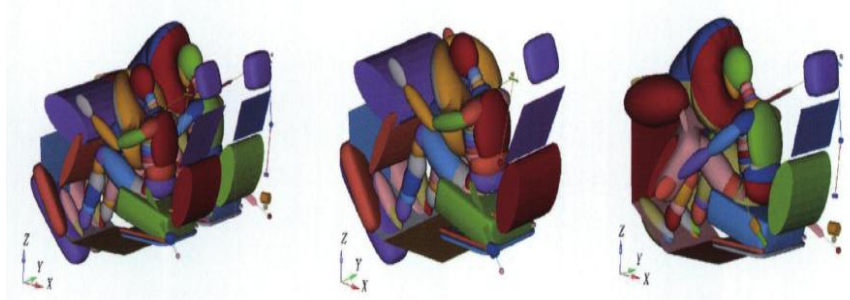

Figure 3. Computer simulation

The energy absorbing space in front of automobile is often limited, so the research and development of materials with high energy absorption efficiency has become the focus of research. Some of the energy absorbing efficiency of higher materials have developed PC/PET materials such as GE, Dow STANDFOAM materials, Tsinghua University and other units are also committed to the development of some new energy absorbing materials, such as solid-liquid composite based on nano porous materials ${ }^{[4]}$.

A parametric human finite element model is developed by University of Michigan and other units, which can significantly improve the development efficiency of a variety of human models ${ }^{[5]}$. The main idea is based on different characteristics (age, weight and height) of the parametric shape and size of the skeleton, derived from human body model of non standard finite element model standard by radial basis function grid changes, to obtain the characteristic parameters (age, weight, height) finite element model of the corresponding the crowd. It has laid a good foundation for the development of automotive safety technology, and also developed adaptive occupant protection system for various populations and working conditions in the future, so as to cope with various situations of real traffic accidents.

In addition, the study of the driver's attention has also made progress. Scholars have divided the driver's distraction into cognitive distraction and visual distraction, the former refers to the driver's cognitive thinking is occupied by other things (such as voice dialogue), the latter refers to the driver did not focus on the road environment (when using a cell phone or navigation system). The research shows that distraction will significantly increase the driver's workload, make it change the vehicle operating behavior, response time becomes longer, the accident rate increased. For example,
Volvo Trucks proposed a solution to monitor the driver's eye movement through the camera to determine whether the driver is distracted, and use the sound and LED lights to provide different levels of reminders. Toyota also uses the camera to observe the driver's head movements in its Pre-crash Safety System, and measure the distance between the upper and lower eyelids to determine whether the driver is dozing.

Some achievements have been made in the development of active safety technology in China. The THMR-V intelligent vehicle developed by Tsinghua University is equipped with CCD camera and navigation system to realize the intelligent driving function. Geely Automobile put forward the concept of GTMS safety development, in order to reduce the casualties of traffic accidents as the development goal, combined with electronic stability control system, adaptive cruise system, front / rear collision warning system and G-Netlink system in one. The safety protection of occupants before collision, collision and collision is realized.In addition, some domestic universities have studied car collisions. The research team of Tsinghua University, Huang Shilin, Zhou Qing and Zhang Jinhuan, carried out a structural transformation design for a van, and tested the crashworthiness of vehicle structure through vehicle crash simulation calculation. Jilin University Lin Yi's research team conducted a structural improvement design and restraint system optimization of the FAW CA6350 van and evaluated the vehicle crashworthiness. Zhang Junyuan independently studied the integrated child restraint system and developed a one-piece child seat to protect the safety of children occupants. Chongqing University Li Xiaohong's team conducted a technical transformation of the Chang'an SC6370 Van and improved the passive safety performance of the vehicle. Hunan University President Zhong Zhihua's research team made technical improvements to the Liuqi N1 Car, set up an airbag research center and developed a variety of car crash occupant protection technology and equipment.

\section{Automotive Safety Technology}

Karl Benz, a famous German engineer, invented the first car in history in 1886 and so far the reform and development of the automobile industry has lasted for nearly 130 years. In the meantime, automobiles have spread to all parts of the world and have rapidly penetrated into the social economy and people's daily life, and have become the most important and most common means of transportation for people. However, in recent years, due to the rapid popularization of automobiles, traffic safety has become more and more serious. The recent "World Disaster Report" pointed out that about 1.3 million people died in traffic accidents each year, and the number of injuries is up to 30 million. The world lost as much as $\$ 3,000$ billion due to traffic accidents, of which up to two-thirds were lost in developing countries. Therefore, car safety performance is of great significance to occupant safety. 
The R \& D of automotive safety technology mainly starting from the whole, the first is the most important thing is to ensure the vehicle safety and comfortable situation as far as possible to prevent the occurrence of traffic accidents, the second is when the vehicle traffic accidents, to minimize the damage caused to the people in the car. At present, automobile safety is divided into two categories: active safety and passive safety.

\subsection{Active safety technology}

Active safety technology means the reasonable design of automobile and scientific structure in the automotive R \& $\mathrm{D}$ and production process, using the very advanced technology in automobile industry, as far as possible to avoid or reduce the vehicle in normal driving sudden collision accidents, to improve the safety of their performance, to reduce the risk of a traffic accident then, to reduce the losses caused by the occupant. In recent years, active safety technology has become an important research object of $\mathrm{R} \& \mathrm{D}$ institutions of major automotive companies.

\subsubsection{Chassis active control technology}

Vehicle active safety control is usually realized by active safety control of chassis. Electronic brake control is one of the most widely used technologies of chassis active control technology. Electronic stability control system (ESC) is considered to be one of the signs of vehicle active safety technology development, by comparing the running state of driver's intention and the actual vehicle system, reasonable distribution of longitudinal and lateral tire force, dynamic behavior and precise control of the vehicle, the driver will try to follow the road, reducing the probability of traffic accident the. According to the U.S NHTSA statistics, ESC can reduce $34 \%$ of bicycle accidents and $71 \%$ of rollover accidents; for SUV, it can reduce $59 \%$ of bicycle accidents and $84 \%$ of rollover accidents[6].

\subsubsection{Security early warning technology}

The security warning technology detects the road environment information through the ultrasonic, vision and radar sensors, and analyzes the various information to remind and warn the driver, thereby reducing the occurrence of accidents. At present, the technology mainly through the use of visual sensors in the car, to detect the surrounding environment, auxiliary driver operation. With the development of vision technology, the security warning system based on monocular vision sensor will be more widely used in vehicles, and data fusion with other sensors will further improve the security of security warning technology.

\subsection{Passive safety technology}

Passive vehicle safety technology refers to the automobile after the collision, using the protection devices to protect the people inside and outside the automobile as much as possible, to minimize damage. Today, most of the car mounted occupant protection devices include anti-collision body, pre tightening seat belts and airbags and so on.

\subsection{Safety belt}

The seat belt was invented by Chaire L. Strath in 1935. It has been used as a must for automobiles. Seat belts are the most representative and major vehicle protection devices in the history of automotive passive safety system's research and development. In the process of automobile traffic accidents, it mainly uses the occupant restraint to absorb and buffer most of the energy generated by collision, so as to avoid the occupants hit into the cockpit or the happening of second collision, as much as possible to reduce occupant injuries and economic losses. According to NHTSA estimates, the use of seat belts reduced $45 \%$ of fatal injuries and $50 \%$ moderate to severe injuries to car front occupants, while $60 \%$ and $65 \%$ for light truck occupants. It saved the lives of 12802 people in the United States in 2014[7-8]. The use of seat belts is shown in Figure 4.

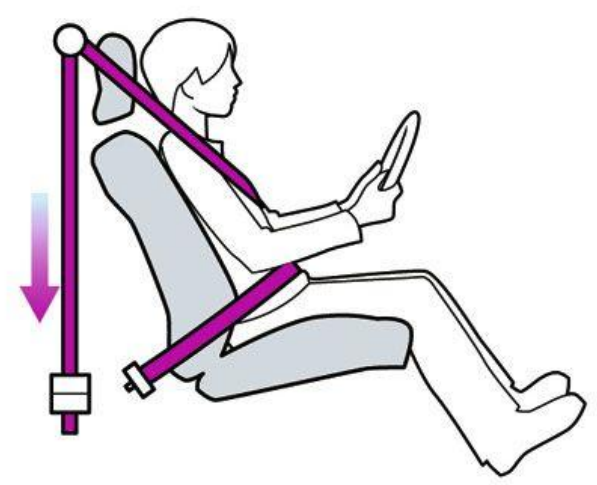

Figure 4. Proper use of seat belts

\subsubsection{Airbag}

Airbags were first invented by the Swedes. The first patent for American airbags was made in the 1950s, and some factories started to develop airbags in the 1970s. In 1972, General Motors took the lead in large-scale airbag field test. After the $1980 \mathrm{~s}$, more cars equipped with airbags, airbag technology tended to improve. In the 1990s, the number of airbag installations increased rapidly and there were many new technology products. The first airbag to prevent side impact was first developed by General Motors in 1996. In the 21 st century the airbag has become a very common and vital safety device in automobiles.

The airbag is an auxiliary occupant restraint system, which works with the seat belt to prevent occupants from being hurt by the car's interior trim. It mainly includes the controller, sensor, gas generator, airbag bag, warning lamp and other components. After the controller receives 
the signal, it immediately begins to analyze and judge whether to open the bag. It will immediately to send the ignition instructions if it is necessary. After receiving the ignition command, generator quickly generate enough gas to air filled gas until the airbags fully open now, and then contact with the soft passenger airbag, preventing the occurrence of two collision and car parts, reducing the traffic accidents caused by the occupant injuries and economic losses. The working principle is shown in Figure5.The startig airbag is shown in Figure 6.

Sensor: One of the most important components of a car's airbag, used to capture signals such as speed and acceleration when a car collides, and send the signal to the controller.

Controller: Used to receive and analyze the speed or acceleration signal of the vehicle, and determine whether to send the ignition signal according to the analysis. In the case of collision, the more accurate judgment of the controller, and can accurately control the ignition time, the more the airbag can be opened correctly, to maximize the protection of the occupant.

Gas generator: When the car collides, the gas generator quickly generates enough gas and immediately inflates to the airbag. The gas can not be harmful to the human body, no pollution to the air, and the gas temperature not too high to avoid burning the human body. At the same time, it requires high stability and high reliability.

Airbag bag: when the airbag expands, the explosive force is large and the temperature is high, which requires the airbag bag of high strength, good elasticity, thermal performance is strong, at the same time with high stability, aging resistance, small volume after folding, and can quickly expand, and the air bag can not scratch the human face.

Among them, the gas generator is the main component of the airbag system, its role is to provide sufficient gas with explosive speed in a very short period of time. When the occupant is in contact with the airbag, the airbag hold up the occupant's head, to avoid touching other parts and reduce the injury.

At present, the typical products are active pop-up hood and pedestrian protection airbags. The active popup cover is triggered by a warning sensor installed on the bumper and other locations. When the vehicle collides, the hood is popped up by gunpowder or spring to increase the cushioning space to reduce the head and upper limb injury. The technology has been applied in the Jaguar XJ, Citroen C6 and other models. The installation of outer airbag in the car's head also appeared in the Ford, Fiat Stilo, Toyota and other models. The hood airbag developed by Ford in 2007 was mounted above the bumper and up to the hood surface. After that, Toyota launched a 360 degree airbag protection system, between the bumper and the engine cover, between the engine cover and the front windshield, the fender, the rearview mirror were installed five airbags to the front all wrapped, thereby enhancing the pedestrian protection effect.

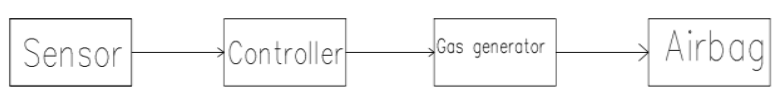

Figure 5. Working principle of airbag

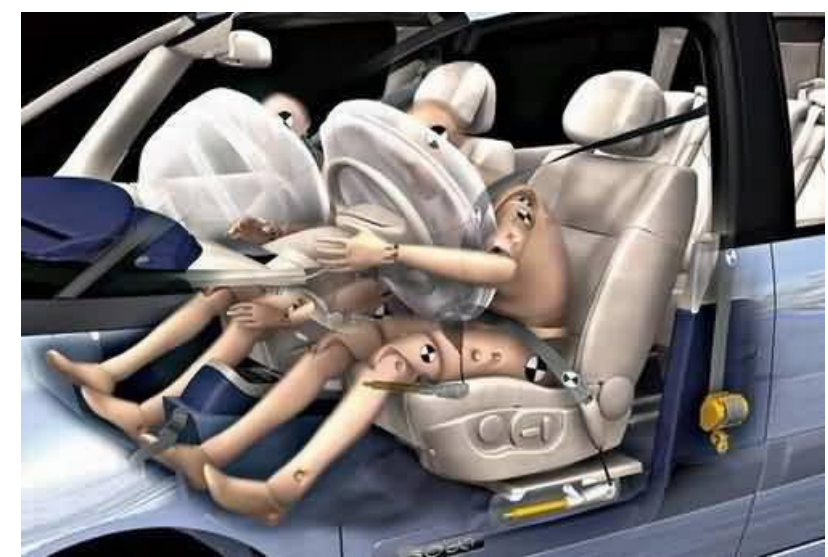

Figure 6. The startig airbag

\section{New airbag system}

At present, the traffic accidents in bad weather have attracted people's attention, and also exposed the problems in the safety of the car. From the data of deaths from various types of car accidents in China in 2004, turnover accounted for about $60 \%$, while the car fall accounted for about $20 \%$, the collision object fell to $10 \% \sim 20 \%$. Among them, the death rate of car crashes increased year by year, and the mortality rate was the highest among the bicycle accidents. The US NHTSA statistics show that rollover accidents are a major form of fatal accidents. In 2014, the number of passengers killed by rollover in the United States accounted for 33\% of the total number of fatalities[9]. On the other hand, the city flood disaster in recent years in sudden extreme rainstorms caused by frequent, which caused a large number of cars were flooded, vehicle floating or sliding instability, and caused some people drowned. In many traffic accidents, collision accidents account for a large proportion (accounting for about 70\%). Collision accidents often lead to economic losses and accident disputes, and even lead to casualties. But at present, when most vehicles collide, only the airbag inside the vehicle is opened, the side protection device is very few, and it can not reduce the damage caused by collision.

Therefore, this paper improves the traditional airbag to reduce the economic losses and casualties caused by traffic accidents and bad rainstorm environment.

\subsection{Basic constitution}

The new airbag system mainly includes the controller, sensor, gas generator, airbag bag, warning lamp and other components. In addition, the airbag system including cab 
safety gasbag, set in front of the car rear airbags and set up in the floor and roof of the car body airbags, each equipped with airbag airbag triggering device the pressure sensor; and a start switch are respectively connected with the input end of the controller, the output terminal of the controller and personal safety airbag, airbag and airbag connecting body collision airbag triggering device. In the storm, the airbag is located in the bottom can timely use buoyancy to make the car floating in the water, convenient personnel escape, to avoid drowning personnel. The airbag around the roof and the roof can be started when the car is hit or overturned, reducing the impact and protecting the safety of the people in the car. Install 4 parachute packs on the four corners of the roof to deal with dangerous situations like falling. Installing 4 parachute packs on the four corners of the roof, to deal with dangerous situations like falling. The canopy should be for textile materials, light weight, good elasticity, good chemical stability, the umbrella rope woven rope or hollow core requirements, compact structure, soft, good elasticity, unevenness of elongation is small. The umbrella line is the suture zone, the parachute, rope connecting material to all parts of the requirements high strength, good smoothness and uniform twist stability. In addition, device should also be parachute bag is fixed in the roof, and trigger to open it when necessary.

\subsection{Working mode}

The working principle of the new airbag system is shown in figure 7.

It is found that the greater the water level is, the greater the force required to escape the door when escaping. Therefore, the shorter the escape time is, the greater the probability of success is. When water depth is $70-80 \mathrm{~cm}$ away from the ground, it is the critical depth of vehicle escape. Therefore, in the rainstorm environment, the water level rises rapidly, and the judgment of water level is the key. The airbag system installs pressure sensors at the bottom of the car to detect the water depth.

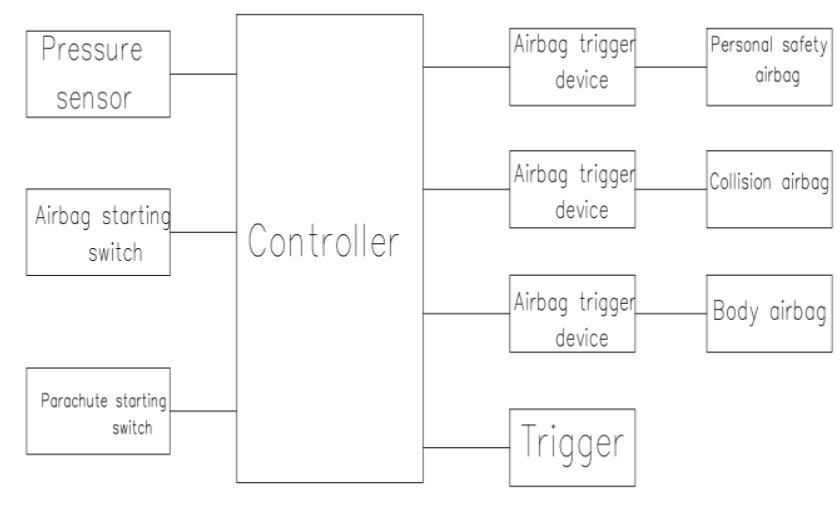

Figure 7. New airbag system working principle

When the water level is higher than the critical water depth, the car bottom and front and rear side airbags are automatically started to protect the personnel safety.
When the car is in danger of collision, that is, when the impact force (deceleration) exceeds the set value, the signal is produced. After starting the system, the four positions airbag is inflated, and the crew airbag system also works.

In addition, when the car has a rollover or falling danger, the system senses the inclination parameters of the vehicle through the vehicle interior attitude sensor. When the angle reaches the critical point, the starting signal is generated. All the airbags inflate and release the four angles parachute, which reduces the impact force of the car landing, and protects the personnel safety as much as possible.

To sum up, the airbag device has a variety of starting methods, including water pressure passive start mode, the active start mode of rainstorm, traffic accident initiation mode, high-altitude falling mode, etc., to adapt to the dangerous situation of the car in different environmental conditions

Water pressure passive start mode: when the vehicle is immersed in water for a certain depth, water pressure reaches a critical value, the water pressure sensor transmits a signal to the MCU, MCU control car airbag, car using buoyancy to float in the water.

The active starting mode of the rainstorm: when the rain enters the car and the car has people or someone finds that the car has been immersed in danger, it starts. The starting system is the radio remote start system. After starting the system, the airbag system of the car bottom and the system of the four position airbag begin to work

The traffic accident start mode: when the car driving start system in a relatively flat area of traffic accident. The collision analysis control is connected to the gate element to control the system. Analysis of the essence of control when the collision impact force (deceleration) signal exceeds the set value, the system. After the start of a four airbag occupant work the airbag system is working.

Falling mode: when the automobile from the start when the parachute into high altitude, with the sensor control and gate components. Essence of sensor control by attitude sensor vehicle status, exceeds the critical value generated signal. After the system starts in four directions and start the air bag and the vehicle airbag, passenger airbag system work.

\section{4 conclusion}

Safety is the eternal theme of automobile technology development. With the development of society and economy, Car ownership keeps increasing; the urbanization promotes the change of urban and rural traffic environment; and the problem of automobile safety becomes more and more serious. In this paper, the current automotive safety technology is summarized; in addition, in view of the bad weather vehicle safety problems, a new airbag system is proposed, and its working mode is described. The effect of the new air bag needs to be tested by experiments. In the future, its' safety will be analyzed by the way of physical simulation. It means how this air bag copes with the environment of rainstorm and the roller accidents which lead to high fall accidents, will be analyzed. Then, further improvements will be made according to the analysis results. 


\section{References}

1. World Health Organization. World report on road traffic injury prevention [R]. Geneva: WHO, 2004. (in Chinese)

2. WANG Xiaojing. The review of ITS for traffic safety [C]// 2008 Fourth China Intelligent Transport Annual Meeting Thesis, 2008-09. (in Chinese)

3. Wiezbicki,T.,Recke.L.,Abramowicz.W.,Gholami.T.,J .Huang.Stress Profiles in Thin-Walled Prismatic Columns Subjected to Crush Loading11.Bending,Computers\&Structures Vol.51No.6,P625-64 1.

4. SUN Yueting, XU Xiaoqing, XU Chengliang, et al. Elastomeric cellular structure enhanced by compressible liquid filler [J]. Sci Rep, 2016, 6: 26694.

5. SHI Xiangnan, CAO Libo, Reed M P, et al. Effects of obesity on occupant responses in frontal crashes: a simulation analysis using human body models [J].
Comput Methods Biomech Biomed Eng, 2015, 18: 1280-1292

6. NHTSA-200727662, Federal motor vehicle safety standards; electronic stability control systems; controls and displays [S]. Washington DC: National Highway Traffic Safety Administration, 2007

7. National Center for Statistics and Analysis. Lives saved in 2014 by restraint use and minimumdrinking-age laws, DOT HS $812 \quad 218$ [R]. Washington, DC: National Highway Traffic Safety Administration, 2015

8. Kahane C J. Lives saved by vehicle safety technologieand associated Federal Motor Vehicle Safety Standard1960 to 2012, DOT HS 812069 [R]. Washington, DC:

9. National Center for Statistics and Analysis. Passenger vehicles: 2014 data (Traffic safety facts), DOT HS 812302 [R]. Washington, DC: National Highway Traffic Safety Administration, 2016. 